\title{
Política comercial exterior de la Unión Europea y las sanciones a Rusia
}

\author{
Antonio Sánchez Andrés \\ Universitat de València
}

\section{Resumen}

En 2014 la Unión Europea comenzó a aplicar unas sanciones contra Rusia. Este trabajo analiza el impacto comercial de estas medidas de política económica. El análisis concentra su atención en las importaciones y las exportaciones mutuas. Finalmente se extraen conclusiones acerca de los límites de las sanciones a corto y largo plazo.

Palabras clave: comercio, economía internacional, Rusia, UE.

Clasificación JEL: F1, F14, P3.

\section{Abstract}

In 2014 the EU began to apply some sanctions against Russia. This paper analyzes the commercial impact of these policy measures. The analysis pay attention on mutual imports and exports. Finally conclusions are drawn about the limits of sanctions in the short and long term.

Keywords: trade, international economy, Russia, EU.

JEL clasification: $F 1, F 14, P 3$.

\section{Introducción}

A finales de 2013 se fue culminando un proceso de acercamiento entre Ucrania y la Unión Europea (UE), que provocó una división interna dentro de ese país centroeuropeo. A principios de 2014 se acrecentaron los conflictos, que condujeron a una ruptura del país. Crimea pasó a integrarse dentro de Rusia y las regiones de Donetsk y Lugansk se independizaron de la administración de Kiev. La Unión Europea y Estados Unidos rechazaron la conducta de Rusia en el conflicto ucraniano y fueron aplicando medidas restrictivas contra Rusia.

En este trabajo se analizará el impacto de las sanciones de la UE como una faceta de su política comercial. Este ámbito es donde se reflejan los efectos más importantes de esas medidas. En primer lugar, se pondrá de manifiesto la importancia del comercio entre la UE y Rusia; posteriormente, se analizará progresivamente el impacto en el ámbito de las exportaciones y de las importaciones, y finalmente se extraerán unas conclusiones sobre las repercusiones de estas medidas de política comercial. 


\section{La nueva política comercial hacia Rusia: las sanciones y su contexto comercial}

Las sanciones de la UE hacia Rusia comenzaron a aplicarse en marzo de 2014, en gran medida motivadas por la presión ejercida sobre Bruselas por Estados Unidos. Estas tuvieron inicialmente un carácter más político, bien de tipo general, como la suspensión de encuentros políticos mutuos, de negociaciones bilaterales sobre visados o de renovación del Acuerdo de Asociación UE-Rusia; bien de tipo específico, como la prohibición de otorgar visados a ciertas personas públicas rusas, así como la inmovilización de sus activos (también de personas jurídicas). Estas medidas se fueron acrecentando hasta entrar en el ámbito más económico y configurarse en nuevos instrumentos de política comercial.

Dentro de las sanciones se encuentra la prohibición en la venta de armamento (también la importación) a Rusia, así como de equipos y tecnologías de doble uso con fines militares, pero también es importante la relacionada con la exportación de ciertas tecnologías para la prospección y producción, en especial, de petróleo. Debe apuntarse también por su trascendencia los límites al acceso a los mercados primarios y secundarios de capitales de la UE para las cinco mayores entidades financieras rusas con participación estatal, así como a varias compañías de hidrocarburos y otras relacionadas con el sector de defensa. Finalmente, con menor impacto relativo, se encuentran los límites a la financiación a través del Banco Europeo de Inversiones y del Banco Europeo para la Reconstrucción y el Desarrollo, así como la suspensión de algunos programas de cooperación.

Cabe señalar que, a pesar de las presiones políticas de Washington, la UE ha excluido la extensión masiva de las restricciones a las exportaciones e importaciones con Rusia. En particular, han quedado fuera de las sanciones las compras de productos energéticos (gas y petróleo) (Consejo de la Unión Europea, 2016).

Este paquete de medidas tiene asociado unos efectos laterales derivados de la respuesta rusa. Moscú decidió aplicar una respuesta asimétrica. La idea general era reaccionar, pero considerando que afectase a sus intereses internos en el menor grado posible. En concreto, se prohibió la importación de ciertos productos alimentarios (animales y vegetales), con el trasfondo de que estas medidas estimularían la reindustrialización interna. Además, no se afectaron las ventas de otros productos estratégicos, como los energéticos ni otras mercancías con cierta relevancia, como las exportaciones de titanio, aluminio o paladio.

La importancia de las sanciones deben apreciarse en el contexto del valor de las relaciones comerciales de la UE con Rusia (véase Cuadro 1). Después de una tendencia al ascenso, en 2012 el total de comercio mutuo alcanzó un valor de 338,6 miles de millones de euros. En 2013 presentó una disminución hasta registrar los 326,4 miles de millones de euros, momento en que inició una caída continua hasta suponer solo 209,6 miles de millones en 2015. No obstante, debe matizarse que en 2013 parte de la reducción se encuentra asociada a una caída en el comercio exterior general de la UE, mientras que la contracción ulterior se debe a particularidades específicas de 
CUADRO 1

COMERCIO CON RUSIA EN LAS RELACIONES COMERCIALES EXTERIORES

DE LA UE-28

(En millones de euros)

\begin{tabular}{|l|r|r|r|r|r|}
\hline & \multicolumn{1}{|c|}{$\mathbf{2 0 1 1}$} & $\mathbf{2 0 1 2}$ & $\mathbf{2 0 1 3}$ & $\mathbf{2 0 1 4}$ & \multicolumn{1}{c|}{$\mathbf{2 0 1 5}$} \\
\hline $\begin{array}{l}\text { Exportaciones extracomuni- } \\
\text { tarias }\end{array}$ & $1.620 .638,8$ & $1.759 .234,5$ & $1.812 .121,0$ & $1.764 .293,0$ & $1.844 .520,4$ \\
\hline $\begin{array}{l}\text { Importaciones extracomuni- } \\
\text { tarias }\end{array}$ & $1.800 .589,4$ & $1.881 .504,1$ & $1.769 .867,5$ & $1.759 .932,6$ & $1.777 .029,0$ \\
\hline $\begin{array}{l}\text { Total comercio exterior ex- } \\
\text { tracomunitarias (X+M) }\end{array}$ & $3.421 .228,1$ & $3.640 .738,6$ & $3.581 .988,5$ & $3.524 .225,6$ & $3.621 .549,3$ \\
\hline Exportaciones a Rusia & $108.587,4$ & $123.441,2$ & $119.450,9$ & $103.202,6$ & $73.886,8$ \\
\hline Importaciones desde Rusia & $201.327,2$ & $215.118,6$ & $.206 .967,0$ & $182.371,4$ & $135.716,8$ \\
\hline $\begin{array}{l}\text { Total comercio con Rusia } \\
\text { (X+M) }\end{array}$ & $309.914,6$ & $338.559,9$ & $326.417,9$ & $285.573,9$ & $209.603,6$ \\
\hline Tasa cobertura (X/M) (\%) & 53,9 & 57,4 & 57,7 & 56,6 & 54,4 \\
\hline
\end{tabular}

FUENTE: Elaboración propia a partir de Ministerio de Economía y Competitividad.

Rusia, puesto que mientras que en 2014 el comercio extracomunitario cayó en un 1,6 por 100 y en 2015 aumentó en un 2,8 por 100, el relacionado con Rusia se contrajo en ambos años en un 12,5 y un 26,6 por 100 , respectivamente.

Dentro del conjunto de comercio exterior con Rusia se puede destacar que las importaciones de la UE casi han duplicado a las exportaciones. En 2011 la tasa de cobertura comercial fue del 53,9 por 100, relación que fue mejorando hasta 2013, cuando ascendió al 57,7 por 100, pero a partir de ese momento empeoró y en 2015 alcanzó el 54,5 por 100 .

Quizá una de las particularidades más relevantes de la aplicación de las sanciones a Rusia se puede extraer a partir de la ubicación de este país dentro del ranking de socios comerciales de la UE (véase Cuadro 2). Durante los tres primeros años del periodo considerado, Rusia ha sido el tercer socio comercial extracomunitario, detrás de Estados Unidos y China, y seguido de Suiza, Turquía, Japón y Noruega. En ese periodo, Rusia concentró algo más del 9 por 100 del comercio exterior de la UE. Este porcentaje es relativamente grande cuando se considera que los dos primeros socios comerciales de la UE concentraban cada uno algo más del 13 por 100 de este tipo de comercio, mientras que Suiza, en términos medios, significó un 7 por 100 y Noruega un 4 por 100. En 2014, la porción del comercio con Rusia cayó al 8,1 por 100, aunque este país siguió ocupando el tercer lugar entre los socios comerciales de la UE, y en 2015 la pérdida fue muy brusca, ocupando Rusia un 5,8 por 100 de la cuota del comercio exterior de la UE, situación que desplazó a este país a la cuarta posición como socio comercial. Así pues, una particularidad importante de las sanciones es que se aplican a un país con el que se tiene una estrecha relación comercial, a diferencia de otros casos cuando se aplicaron este tipo medidas político-económicas. 


\section{CUADRO 2}

PRINCIPALES SOCIOS COMERCIALES DE LA UE-28

(Porcentaje del comercio extracomunitario)

\begin{tabular}{|l|r|r|r|r|r|}
\hline & $\mathbf{2 0 1 1}$ & $\mathbf{2 0 1 2}$ & $\mathbf{2 0 1 3}$ & $\mathbf{2 0 1 4}$ & $\mathbf{2 0 1 5}$ \\
\hline Estados Unidos & 13,3 & 13,7 & 13,5 & 14,8 & 17,1 \\
\hline China & 13,8 & 13,2 & 13,2 & 14,5 & 15,8 \\
\hline Rusia & $\mathbf{9 , 0}$ & $\mathbf{9 , 3}$ & $\mathbf{9 , 1}$ & $\mathbf{8 , 1}$ & $\mathbf{5 , 8}$ \\
\hline Suiza & 6,9 & 6,6 & 7,4 & 6,7 & 7,0 \\
\hline Noruega & 4,1 & 4,1 & 3,9 & 3,8 & 3,4 \\
\hline Turquía & 3,5 & 3,4 & 3,5 & 3,6 & 3,8 \\
\hline Japón & 3,5 & 3,3 & 3,1 & 3,1 & 3,2 \\
\hline
\end{tabular}

NOTA: La información para China incorpora la correspondiente a China continental, Hong Kong y Macao.

FUENTE: Elaboración propia a partir de Ministerio de Economía y Competitividad.

Finalmente, cabe señalar que la Unión Europea constituye el primer socio comercial de Rusia. De hecho, utilizando fuentes del Servicio de Aduanas de la Federación Rusa (Fyedyeral'naya Tamozhyennaya Sluzhba), se pone de manifiesto que, en 2011, el 48,1 por 100 del comercio de Rusia se orientaba hacia la UE. Este porcentaje fue ascendiendo hasta 2013, cuando alcanzó el 49,5 por 100. En el siguiente año se redujo al 48,0 y en 2015 era del 44,8 por 100 . Debe destacarse que este comercio se distribuye muy heterogéneamente, puesto que seis países concentran más del 62 por 100 del comercio mutuo Rusia-UE (Cuadro 3).

\section{CUADRO 3}

RUSIA Y EL COMERCIO GLOBAL CON LOS PRINCIPALES SOCIOS DE LA UE

\begin{tabular}{|l|c|r|r|r|r|r|}
\hline & $\begin{array}{c}\text { Porcentaje } \\
\text { total } \\
\text { comercio }\end{array}$ & \multicolumn{7}{|c|}{ Tasas de crecimiento } \\
\hline & $\mathbf{2 0 1 1 - 2 0 1 5}$ & $\mathbf{2 0 1 1}$ & $\mathbf{2 0 1 2}$ & $\mathbf{2 0 1 3}$ & $\mathbf{2 0 1 4}$ & $\mathbf{2 0 1 5}$ \\
\hline Francia & 5,9 & 15,1 & $-0,7$ & $-12,9$ & $-7,0$ & $-35,6$ \\
\hline Países Bajos & 11,0 & 19,4 & 14,4 & $-2,3$ & $-14,3$ & $-28,2$ \\
\hline Alemania & 23,1 & 29,4 & 7,3 & $-3,9$ & $-12,7$ & $-25,4$ \\
\hline Italia & 9,2 & 16,3 & 8,0 & 9,4 & $-13,5$ & $-20,2$ \\
\hline Finlandia & 4,8 & 18,6 & $-1,1$ & $-2,3$ & $-16,6$ & $-31,1$ \\
\hline Polonia & 8,3 & 29,4 & 21,2 & $-8,6$ & $-10,1$ & $-26,3$ \\
\hline Total & 62,3 & & & & & \\
\hline
\end{tabular}

FUENTE: Elaboración propia a partir de Ministerio de Economía y Competitividad. 


\section{Importaciones de la UE desde Rusia}

En 2011 la UE realizó compras en Rusia por valor de 267,6 miles de millones de dólares, cifra que se fue acrecentando hasta 2013, momento en que alcanzó los 282,2 miles de millones de dólares (véase Cuadro 4). A partir de ese año se contrajeron estas compras hasta significar, en 2014, 258,5 miles de millones de dólares y en 2015 los 165,6 miles de millones de dólares. Así pues, existe un crecimiento durante los primeros años del quinquenio considerado hasta que en 2014 se registró un decrecimiento del 8,4 por 100 y un caída en un 35,9 por 100 en 2015. Al observar el comercio según secciones arancelarias, se puede destacar que durante el periodo considerado, dos de ellas agrupan al menos el 87 por 100 de las exportaciones rusas. Se trata de productos minerales y metales. Más aún, el 78 por 100 de las ventas rusas se concentra en la primera de las mencionadas secciones comerciales que, por añadidura, está formada esencialmente por hidrocarburos (petróleo, gas y sus derivados). Respecto a los metales, debe destacarse que se trata de hierro y acero con bajo nivel de valor añadido y, en ciertos casos, cobre, níquel y aluminio. Esta partida ha oscilado entre un 6,4 y un 8,4 por 100 de las exportaciones rusas a la UE y también se ha resentido seriamente en su valor, de hecho, durante los últimos tres años ha ido experimentando un decrecimiento continuado, pasando de registrar 19,6 a 14 mil millones de dólares de 2011 a 2015.

Si se observa el comportamiento del precio del petróleo, en los dos últimos años este cayó en un 8,6 por 100 en 2014 y en un 44,2 por 100 en 2015, magnitudes equivalentes a los descensos en el valor de la partida de exportaciones rusas de productos minerales (y también de las exportaciones en su conjunto). Así pues, se puede avanzar la idea de que la reducción en el valor de las compras de la UE se encuentran asociadas a la caída del precio del petróleo más que a sanciones no explicitadas asociadas a política comercial (véase Cuadro 5). 


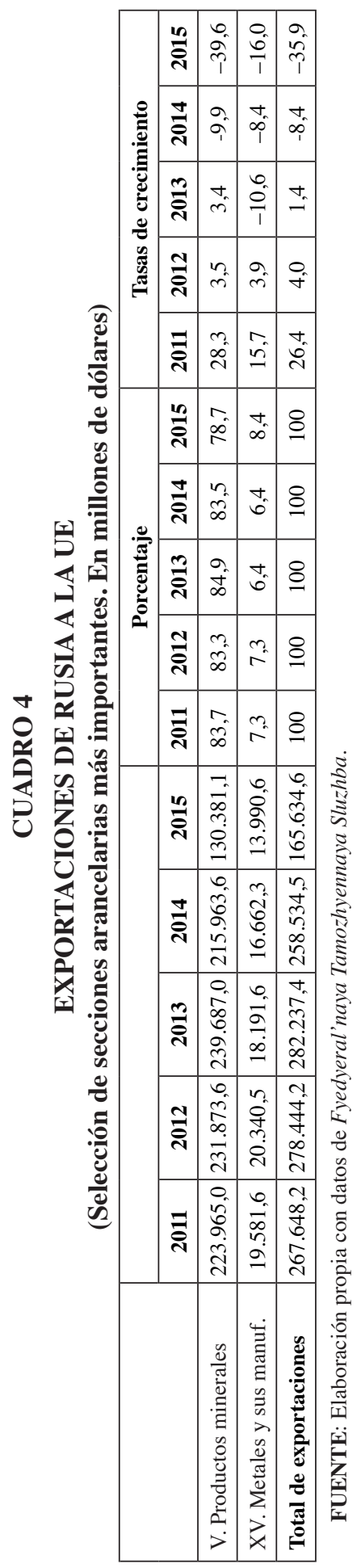




\section{CUADRO 5}

EVOLUCIÓN DEL PRECIO DEL PETRÓLEO

\begin{tabular}{|l|c|r|r|r|r|}
\hline & $\mathbf{2 0 1 1}$ & $\mathbf{2 0 1 2}$ & $\mathbf{2 0 1 3}$ & $\mathbf{2 0 1 4}$ & $\mathbf{2 0 1 5}$ \\
\hline Barril Brent (en dólares) & 110,9 & 111,6 & 108,7 & 99,4 & 55,5 \\
\hline Tasa de crec./decrec. & 38 & 0,6 & $-2,6$ & $-8,6$ & $-44,2$ \\
\hline
\end{tabular}

FUENTE: Elaboración propia con datos de la Secretaría de Economía del Gobierno de México.

Si se analizan más pormenorizadamente las exportaciones de combustibles rusos a la UE, se detectan varias características reseñables. En primer lugar, una fuerte concentración de las ventas rusas en la UE, que alcanzan al menos el 60 por 100 de este tipo de exportaciones. En segundo lugar, más de dos tercios del total de compras de la UE son realizadas por seis países (Alemania, Países Bajos, Italia, Francia, Polonia y Finlandia), que significan más del 40 por 100 de la cuota de mercado rusa de exportaciones de combustibles (Cuadro 6).

\section{CUADRO 6}

COMPRAS DE COMBUSTIBLES EN RUSIA DE LOS SEIS PRINCIPALES SOCIOS DE LA UE (En millones de dólares)

\begin{tabular}{|l|r|r|r|r|r|}
\hline & \multicolumn{1}{|c|}{$\mathbf{2 0 1 1}$} & \multicolumn{1}{c|}{$\mathbf{2 0 1 2}$} & \multicolumn{1}{c|}{$\mathbf{2 0 1 3}$} & \multicolumn{1}{c|}{$\mathbf{2 0 1 4}$} & \multicolumn{1}{c|}{$\mathbf{2 0 1 5}$} \\
\hline Total seis grandes socios UE & $153.035,5$ & $160.734,8$ & $162.169,1$ & $150.241,4$ & $89.535,6$ \\
\hline Porcentaje seis socios/total UE & 68,7 & 69,7 & 68,0 & 69,9 & 69,0 \\
\hline $\begin{array}{l}\text { Porcentaje seis socios/Total ventas } \\
\text { rusa combustibles }\end{array}$ & 42,3 & 43,5 & 43,7 & 43,5 & 41,4 \\
\hline Total UE & $222.633,1$ & $230.717,2$ & $238.605,2$ & $214.789,1$ & $129.674,1$ \\
\hline Porcentaje UE/X Rusia & 61,5 & 62,4 & 64,4 & 62,2 & 60,0 \\
\hline Total Rusia (millones de dólares) & $361.967,1$ & $369.496,5$ & $370.774,5$ & $345.575,7$ & $216.164,1$ \\
\hline
\end{tabular}

NOTA: Los seis principales países son: Alemania, Francia, Finlandia, Italia, Países Bajos y Polonia.

FUENTE: Elaboración propia con datos de Fyedyeral'naya Tamozhyennaya Sluzhba.

Esta concentración conduce a una reflexión adicional acerca de una discusión que se levantó en la segunda mitad de la década anterior. Consistía en que la excesiva dependencia de compras de hidrocarburos de la UE en Rusia podría conducir a un uso político de los recursos energéticos (Sánchez, 2010). Las características expuestas de las exportaciones rusas a la UE ponen de manifiesto que existe más bien una interdependencia. Una prueba de tal situación es que ambas partes han sido exquisitas en la exclusión explícita de los recursos energéticos en sus políticas comerciales restrictivas. 


\section{Las ventas de la UE en Rusia}

Las importaciones rusas desde la UE fueron de 127,6 miles de millones de dólares en 2011 y aumentaron progresivamente hasta alcanzar en 2013 los 134,2 miles de millones de dólares. En el año 2014 se redujeron hasta los 118,4 mil millones de dólares y aún se contrajeron más todavía el año siguiente cuando se registraron ventas de la UE a Rusia por valor de 70,1 miles de millones de dólares. Así pues, en 2014 el decrecimiento en las compras rusas a la UE fue de 11,8 por 100, mientras que en 2015 la disminución alcanzó un 40,7 por 100 (véase Cuadro 7).

Las ventas de la UE a Rusia se encuentran más diversificadas que en el sentido inverso. De hecho, existen seis secciones arancelarias en las que las importaciones de Rusia presentan una cuota sobre el total superior al 5 por 100 del total.

En primer lugar se encuentran las ventas de maquinarias mecánicas y aparatos eléctricos, que han significado durante el quinquenio considerado alrededor de un 30 por 100 del total de las exportaciones de la UE a Rusia. En 2011, estas ventas registraron 38,2 miles de millones de dólares y fueron ascendiendo durante los dos años siguientes y solo en 2014 disminuyeron las exportaciones hasta alcanzar un nivel similar al de cuatro años antes. En 2015, esta partida experimentó una reducción en el 40 por 100, dejándola en 22,8 miles de millones de dólares.

En segundo lugar aparecen dos secciones arancelarias: los productos químicos y los materiales de transporte. En los productos químicos se parte de unos 20 mil millones de ventas a Rusia en 2011, que aumentaron continuamente hasta que tuvo lugar una primera contracción en 2014 equivalente al 9,7 por 100 y otra más fuerte en 2015 del 30,3 por 100. En el caso de los materiales de transporte, casi la mitad está constituido por embarcaciones y el resto por vehículos, pero también, en ciertos casos, por material ferroviario. Este último tipo de productos ha ido perdiendo cuota de mercado progresivamente.

En tercer lugar destacan las secciones arancelarias de plásticos y de metales. Respecto a la primera, ha experimentado una caída, como en el resto de partidas, pero proporcionalmente ha sido menor, de manera que en los dos últimos años ha ganado porcentaje en su peso dentro de las importaciones rusas. En cuanto a la segunda sección de importaciones, consisten, al igual que en las exportaciones, en productos de hierro y acero con bajo nivel de valor añadido, completados con algunos de aluminio y mercancías metálicas con grado de elaboración.

Para explicar esta evolución tan negativa en los dos últimos años, se ha de recurrir a factores económicos que han impactado en la economía rusa. Como consecuencia de la caída en el precio del petróleo ha tenido lugar una menor transformación de divisas en rublos, que se ha reflejado en una depreciación del rublo (véase Cuadro 8). A título de referencia, en términos medios durante el año 2013, un dólar americano se intercambio por 42,3 rublos y, en 2014, por 41,7 rublos. Respecto a este último año cabe señalar que hubo una cierta depreciación del rublo que culminó de forma abrupta en la parte final: mientras que en el tercer trimestre del año 2014, 36,7 rublos equivalían a un dólar, en el último trimestre la moneda americana se cotizó a 59,4 rublos. Esa tendencia 


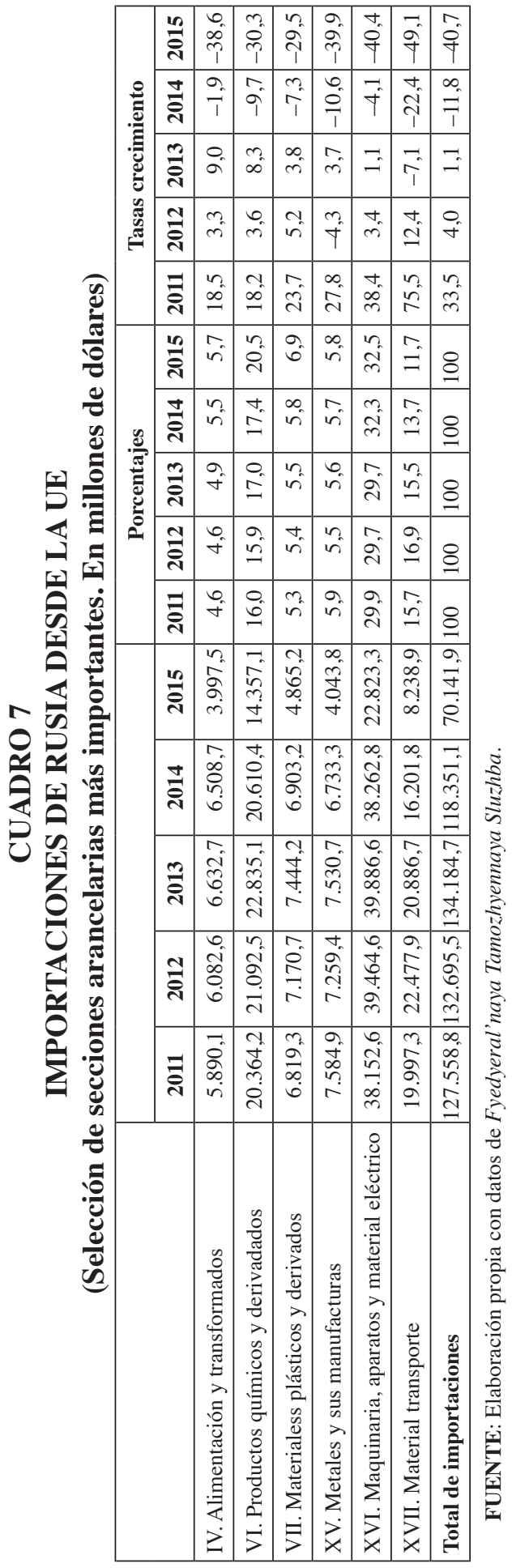




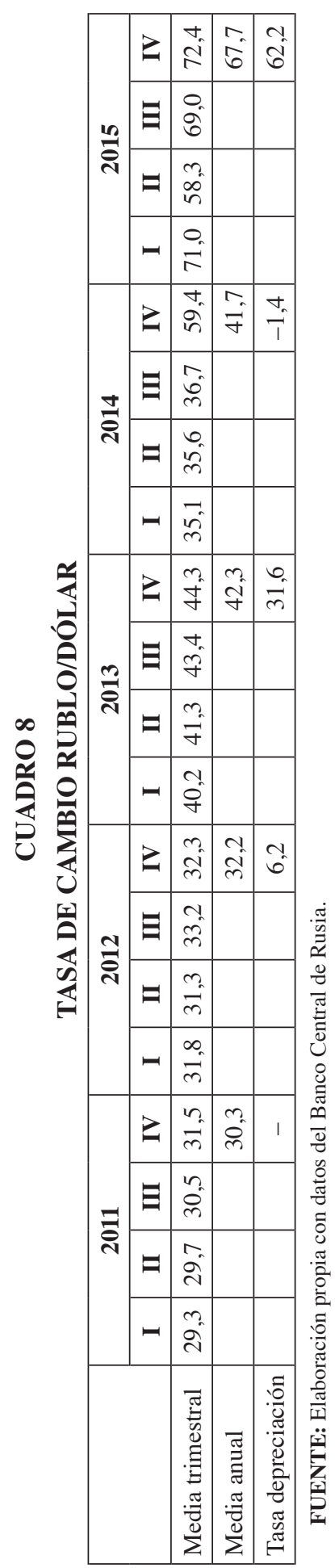


a la depreciación se mantuvo durante el siguiente año cuando en términos medios un dólar americano costó 67,7 rublos. Es decir, en términos medios en 2015 el rublo se depreció en un 60 por 100. Así pues, desde finales de 2014, las importaciones rusas se transformaron en, al menos, un 60 por 100 más caras, con el consiguiente impacto negativo sobre la demanda.

Sin embargo, debe destacarse un factor adicional interno. Este se trata de la ralentización en la dinámica de crecimiento de la economía rusa (Cuadro 9). Después del fuerte impacto negativo del shock de 2008-2009, la economía rusa creció en 2010 a un ritmo del 4,5 por 100; sin embargo, en el siguiente año aumentó un 4,3 por 100 y en 2012 se acrecentó su tamaño en un 3,4 por 100. Esta tendencia a la extinción en la capacidad de crecimiento quedó constatada en 2013 cuando aumentó la economía rusa un 1,3 por 100. En 2014 coincidió la caída de precio del petróleo y las sanciones, pero como los contratos comerciales estaban en marcha, en sí, aunque pudiesen suponer un impacto negativo, una parte significativa de la incapacidad de crecimiento se puede atribuir a los propios límites en la dinámica de la economía rusa. Este freno se reflejó en un caída en las importaciones en 2014, acrecentado en 2015.

\section{CUADRO 9}

CRECIMIENTO ECONÓMICO EN RUSIA

\begin{tabular}{|c|c|c|c|c|c|c|c|}
\hline & 2009 & 2010 & 2011 & 2012 & 2013 & 2014 & 2015 \\
\hline PIB, miles de millones dólares & $1.222,6$ & $1.525,4$ & $1.904,9$ & $2.000,2$ & $2.079,0$ & $1.860,6$ & $1.415,4$ \\
\hline Tasa de crecimiento real & $-7,1$ & 4,5 & 4,3 & 3,4 & 1,3 & 0,6 & $-3,5$ \\
\hline
\end{tabular}

FUENTE: Elaboración propia con datos de Economist Intelligence Unit.

Una mención a parte requieren las importaciones de productos animales y vegetales objeto de contrasanciones rusas (véase Cuadro 10). En cuanto a las importaciones rusas de productos animales, en 2014 cayeron en un 62,2 por 100 y en 2015 en un 76,8 por 100. En cuanto a los productos vegetales, la disminución en las importaciones fue del 29,5 y 61,2 por 100 en los dos años mencionados, respectivamente. Para realizar una aproximación del impacto real de las contrasanciones rusas sobre la UE, debe señalarse que los productos elaborados y de tipo graso han sido afectados de manera más residual. Por este motivo se puede asumir, en cierta medida, que las caídas en las secciones arancelarias III (productos grasos) y IV (transformados alimentarios) podrían explicarse mayoritariamente por la depreciación de la moneda y la ralentización económica del país. Debe reconocerse que, en ciertos casos, estos productos también se vieron afectados por las contrasanciones y, por tanto, el punto de referencia dibujado marcaría una estimación mínima de las medidas rusas de respuesta a las de la UE. En particular, como el decrecimiento en estas dos partidas mencionadas en 2014 y 2015 fue del 1,4 y del 38,3 por 100, respectivamente, se podría asumir que estos porcentajes serían aproximadamente representativos del impacto de la evolución del 


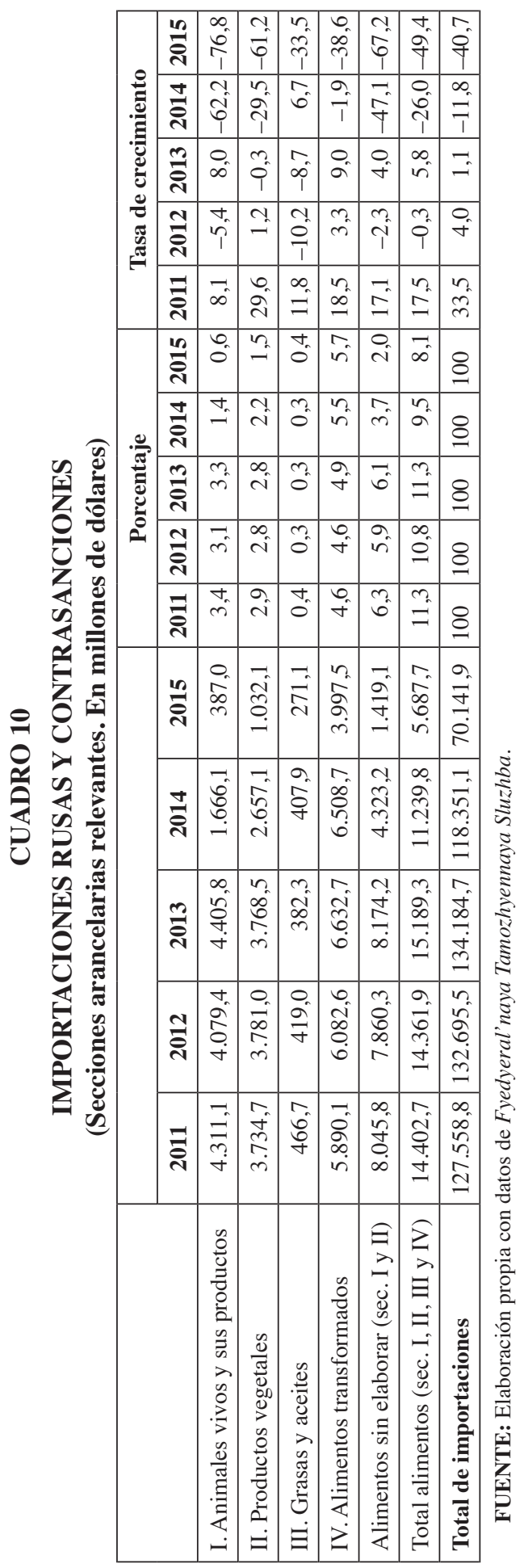


rublo y de la economía rusa, y el resto del nivel del decrecimiento en las importaciones de productos animales y vegetales se derivarían de las contra-sanciones. Así pues, las contrasanciones habrían tenido un impacto en 2014, de 4,4 miles de millones de dólares y en 2015 de 3,1 miles de millones de dólares. Otra valoración sería atribuir a las contrasanciones la reducción en importaciones desde antes de la entrada en vigor de este tipo de política comercial. Esta explicación se justificaría en que estos productos, independiente de la evolución de la economía rusa, se habrían visto afectados por las restricciones. Como en 2013 se alcanzaron unas importaciones de 8,1 miles de millones de dólares en las secciones arancelarias I y II, mientras que en los dos siguientes años se registraron, respectivamente, valores de 4,3 y 1,4 miles de millones de dólares, entonces la diferencia (impacto de las contra-sanciones) sería en 2014 de 3,8 miles de millones de dólares y en 2015 de 6,8 miles de millones de dólares. Por consiguiente, se puede establecer a título de referencia que las contrasanciones rusas tendrían un coste que oscilaría entre 7,5 y 10,6 miles de millones de dólares.

Además de este efecto cuantitativo negativo para la UE debe ponerse de manifiesto una implicación cualitativa. Las pérdidas en estos mercados rusos significa la ruptura en contactos comerciales y empresariales que difícilmente pueden reconstituirse, al tiempo que, en gran medida, han supuesto una desviación de comercio desde la UE a otros países, como Argentina o India. La reconstitución de lazos comerciales y la aparición y consolidación de nuevos competidores supone una dificultad adicional para conseguir recuperar en el futuro este mercado, si se eliminan las restricciones comerciales.

\section{Conclusiones}

Dentro de la política comercial exterior de la UE existe una faceta a la que se le ha concedido poco importancia. Se trata de la imposición de sanciones. Su aplicación a Rusia en 2014 ha puesto de manifiesto su actualidad. No obstante, su impacto en términos comerciales se puede cuestionar, puesto que ha tenido un efecto muy residual sobre el comercio directo. Esto se debe a que el principal tipo de productos, en especial en exportaciones, es decir, los de carácter energético, han sido excluidos del área de referencia de las sanciones. En cuanto a las ventas a Rusia, la parte más sensible afectaba a los equipamientos petroleros, que pueden repercutir, en ciertos casos, en los de tipo gasistas. En estos casos, simplemente se han desplazado temporalmente los proyectos de inversión y el impacto comercial no parece muy visible, a corto y medio plazo. Debe señalarse que respecto a las importaciones rusas de equipamientos de doble uso con aplicación militar realizados por entidades pertenecientes a la industria de defensa también parece que han tenido un impacto secundario. Adicionalmente, las ventas de armamento de países de la UE a Rusia son prácticamente inexistentes, con alguna excepción (la venta de portahelicópteros de Francia).

La repercusión más destacada de las sanciones, si se extiende temporalmente su aplicación, se observará en la caída en la capacidad productiva en el sector energé- 
tico ruso. Sin embargo, debe matizarse que como cerca de dos tercios de este tipo de productos rusos van destinados a la propia UE, los resultados negativos también acabarán reflejándose en esa área europea.

Quizá uno de los impactos más negativos que está teniendo lugar como resultado de las sanciones es en el ámbito financiero. Las restricciones en crédito están generando una carestía financiera en Rusia, que está frenando los proyectos de inversión y contribuyendo a la reducción en las importaciones. Así pues, desde este punto de vista, las repercusiones comerciales presentan cierta relevancia, aunque con el consiguiente impacto negativo cara la UE.

Un efecto secundario de las sanciones ha sido la respuesta comercial de Rusia. Esta se ha presentado en un espectro relativamente estrecho de productos, a saber, animales y vegetales. El resultado ha sido una reducción en las importaciones de este tipo de producto, con la aspiración de aumentar las respectivas producciones internas, aunque, por el momento, ha generado un desabastecimiento interno, una subida en los precios de los alimentos y un desvío de comercio hacia otros países. En el caso de la UE los resultados negativos se han distribuido de manera desigual entre países y en regiones dentro de estos últimos.

Es decir, aunque a partir de 2014 ha tenido lugar una abrupta contracción en los flujos comerciales mutuos entre la UE y Rusia, esta es explicable solo de manera marginal por las sanciones y, en su mayoría, es la resultante de la crisis productiva interna de Rusia y de la caída en el precio del petróleo, con el consiguiente impacto en la depreciación del rublo. Por añadidura, aquello que está generando las sanciones no es un problema económico, sino otro, más bien, de confianza política, cuyas repercusiones económicas son difíciles de evaluar, y se está menospreciando sus efectos futuros.

\section{Referencias bibliográficas}

[1] BANCO CENTRAL DE RUSIA. Dinamika ofitsial'nogo kursa zadannoy valyutï (Dinámica del tipo de cambio oficial de divisas). Recuperado en junio de 2016 de $h t t p: / / c b r . r u /$ currency_baseldynamics.aspx.

[2] CONSEJO DE LA UNIÓN EUROPEA (2016). Medidas restrictivas en respuesta a la crisis en Ucrania. Recuperado en junio de 2016 de http://www.consilium.europa.eu/es/ policies/sanctions/ukraine-crisis/) (última actualización 20 junio 2016).

[3] MINISTERIO DE ECONOMÍA Y COMPETITIVIDAD. Data Comex: Estadísticas de Comercio Exterior. Recuperado en junio de 2016 de http://datacomex.comercio.es/index. htm).

[4] ECONOMIST INTELLIGENCE UNIT. Russia. Country Report. March, EIU, London (varios años).

[5] FYEDYERAL'NAYA TAMOZHYENNAYA SLUZHBA. Tamozhyennaya statistika vnyeshney torgovli Rossiyskoy Fyedyeratsii (Estadística de Aduanas de comercio exterior de la Federación Rusa). Moskva (varios años). 
[6] SÁNCHEZ ANDRÉS, A. (2010). «Rusia y la política energética de la Unión Europea». Investigaciones Regionales, núm. 17, pp. 165-194.

[7] SECRETARÍA DE ECONOMÍA DEL GOBIERNO DE MÉXICO. Históricos precios diarios petróleo WTI, Brent y MME. Recuperado en junio de 2016 de http://portalweb. sgm.gob.mx/economia/es/energeticos/precios-historicos.html). 